\title{
Optic disc measurement: a comparison of indirect ophthalmoscopic methods
}

\footnotetext{
Department of

Ophthalmology, University Hospital,

Nottingham

A F Spencer

$S$ A Vernon

Correspondence to:

Miss A F Spence

Department of

Ophthalmology, University

Hospital, Nottingham NG7 2UH.

Accepted for publication 17 May 1995
}

\author{
Anne Fiona Spencer, Stephen A Vernon
}

\begin{abstract}
Aims-Two methods of indirect ophthalmoscopic estimation of optic disc size, the 78 dioptre lens and optic disc biometer were evaluated.

Methods-Twenty nine eyes of 29 patients were measured by both methods and compared with optic disc size calculated using the three planimetric corrections described by Bengtsson and Krakau.

Results-The closest agreement with the clinical measurements was found using correction 3. There was a significant difference between both the $78 \mathrm{D}$ lens $(p<0.0001)$ and the biometer $(p=0.0027)$ and the planimetric results. There was also a significant difference between the two clinical methods $(p<0.0001)$. Both methods showed acceptable intraobserver variation (CoV 2.45\% and 3.13\% respectively).

Conclusion-Overall, both methods give larger measurements than planimetry; the $78 \mathrm{D}$ lens by $0.41 \mathrm{~mm}$ and the biometer by $0.15 \mathrm{~mm}$. Neither method gives a satisfactory estimation of optic disc size when compared with planimetry.

(Br f Ophthalmol 1995; 79: 910-915)
\end{abstract}

Clinical assessment of the optic nerve head is of great importance in the glaucoma suspect. Ganglion cell loss due to glaucoma causes both localised and generalised changes in the neuroretinal rim and an increase in the size of the optic cup. ${ }^{1-4}$ These changes, which may precede visual field loss, may be seen on direct ophthalmoscopy or at the slit-lamp biomicroscope with the use of condensing lenses. ${ }^{4-6}$ However, previous studies have demonstrated the variability between expert observers in assessing the optic nerve status. ${ }^{7}$ Optic disc size is known to vary considerably between individuals ${ }^{8} 9$ yet it has been shown that large optic discs are more likely to be classified as glaucomatous and small discs are more likely to be classified as normal on clinical examination. ${ }^{10} \mathrm{~A}$ method of measuring optic disc size accurately in vivo would therefore be of help in assessing whether the optic cup was within normal limits for the size of the optic disc and therefore whether the disc shows glaucomatous damage.

Current methods for in vivo measurement include planimetry ${ }^{811} 12$ and more complex computerised image analysis from stereophotography ${ }^{13-16}$ including the Humphrey retinal analyser ${ }^{17}$ and the Rodenstock optic nerve head analyser. ${ }^{18}$ These methods are time consuming or may require specialist equipment not readily available in the outpatient department. An adaptation of indirect ophthalmoscopy for optic disc measurement has been developed ${ }^{19-21}$ and is now commercially available as the 'optic disc biometer'. More recently, using the same optical principle, the 90 dioptre condensing lens, at the slit-lamp biomicroscope, has been described for the estimation of optic disc size. $^{22}$ The 78 dioptre lens yields a larger image size than the 90 dioptre lens and therefore should be preferable as measurements will not be at the lower limits of the scale on the slitbeam.

The aim of this study was to compare measurements of optic disc size, by measuring vertical disc diameter, obtained with both indirect ophthalmoscopic methods - that is, the optic disc biometer and a 78 dioptre condensing lens, and established planimetric methods. ${ }^{23}$ In addition intraobserver variability was assessed for each of the techniques.

\section{Materials and methods}

Thirty eyes of 30 patients were examined. One patient was unable to tolerate the optic disc biometer measurement and therefore the results from 29 eyes are analysed. There were nine normals, six ocular hypertensives, and 14 glaucoma patients. All the patients had a visual acuity of $6 / 9$ or better in the study eye. Twenty three eyes had a refractive error of up to plus or minus 3 dioptres and six eyes were greater than plus or minus 3 but less than plus or minus 7 dioptres.

One eye of each patient was dilated with tropicamide eyedrops $1 \%$ and vertical disc diameter was measured by two observers in a random order. Both observers were experienced in optic disc measurement from previous studies. ${ }^{24} 25$ The first observer measured disc diameter at the slit-lamp biomicroscope using a 78 dioptre condensing lens. The second observer used the 'optic disc biometer' to measure the vertical disc diameter. The optic disc was defined as the area inside the white peripapillary scleral ring (Elschnig). The vertical diameter was defined as the distance from the edge of the nerve fibre rim at 12 o'clock to the edge of the nerve fibre rim at 6 o'clock.

\section{DIOPTRE LENS MEASUREMENTS}

The Haag-Streit slit-lamp biomicroscope used had been calibrated before the study (see below). The 78 dioptre lens used was manufactured by Volk. The lens has a magnification constant of 0.86 (instruction manual, Volk). 
The optic disc was viewed with the $78 \mathrm{D}$ lens as in a normal clinical examination. The lens was held in front of the patient's eye and the optic disc brought into focus by moving the biomicroscope away from the lens. A narrow vertical slit-beam of light, focused on the surface of the optic disc, was progressively reduced in size from $5 \mathrm{~mm}$ until it was judged to correspond to the size of the disc. The beam height was then recorded, by the second observer, from the scale on the slit-lamp. This was then reset to $5 \mathrm{~mm}$ and the measurement was repeated twice, the first observer was therefore 'blind' to the results. As the slit-lamp beam height scale is calibrated in $0.1 \mathrm{~mm}$, the reading was judged to the nearest $0.05 \mathrm{~mm}$. The mean of the three measurements, adjusted for the magnification factor, was then used in comparison with the other methods of measurement.

\section{OPTIC DISC BIOMETER MEASUREMENTS}

The details of this instrument have been described elsewhere. ${ }^{19-21}$ This adaptation of indirect ophthalmoscopy comprises a 15 dioptre condensing lens within an 'optical spacer' which has a fixation target at the principal plane of the lens. A pair of electronic digitised calipers is then used to make measurements of the optic disc diameter. Three measurements of each patient were taken, the calipers were closed between each reading and the calibration checked (that is, the digitised reading was zero). They were then opened progressively until they were judged to correspond to the vertical disc diameter. When the footswitch was depressed this reading was transferred to a microprocessor unit which automatically calculated the vertical disc diameter. As the measurements seen on the calipers is not the same as the computed optic disc size the observer was also 'blind' to the results obtained. The mean of the three readings was used for comparison with the other methods of measurement.

One patient was unable to cooperate with measurements by the biometer, being unable to tolerate indirect ophthalmoscopy with a dim light. Therefore only 29 eyes were included in these results.

\section{SLIT-BEAM CALIBRATION}

A focused slit-beam of light was projected onto a card on which were printed parallel lines at different distances apart. The distance between five pairs of lines ranging from 1-5 $\mathrm{mm}$ apart was measured with a micrometer screw gauge by two observers, the mean being taken as the true distance. The size of the slitbeam was then adjusted to coincide with the distance between the two lines and this was read off the scale on the biomicroscope in a similar manner to that employed for the disc height. The slit-beam was found to be $0.2 \mathrm{~mm}$ smaller than the scale across the range of 1 to 5 $\mathrm{mm}$ calibrated. The results were adjusted accordingly.

\section{PHOTOGRAPHIC CORRECTIONS}

The vertical disc diameter was calculated from photographic slides using the estimates described by Bengtsson and Krakau. ${ }^{23}$ Photographs of the optic discs were taken at the highest magnification, 30 degree setting, with a Topcon fundus camera. A camera constant for the camera used was calculated by the method described by Bengtsson and Krakau. ${ }^{23}$ The axial length and corneal curvature of the eyes was measured using calibrated instruments (Coopervision ultrascan digital $A$ and Javal Schiotz keratometer) and spectacle refractions were performed by experienced optometric staff at a separate examination. The photographic slides of the optic discs were projected onto a screen and the optic disc vertical diameter (using the same criteria as described above) was measured by two independent observers and the mean reading taken. All three estimates described in Bengtsson and Krakau's paper were then applied to the mean image height obtained - that is, using (a) axial length only, (b) spectacle correction only, and (c) using spectacle correction and keratometry.

\section{ANALYSIS}

The mean of each observer's readings are compared by simple regression analysis with the planimetric results and with each other. The level of agreement is also demonstrated. ${ }^{26}$ The coefficient of variation is calculated for both indirect methods of examination as a measure of intraobserver variation.

\section{Results}

MEASUREMENT OF OPTIC DISC SIZE BY 78 D

LENS AND OPTIC DISC BIOMETER

The range of optic disc size measured, the mean, and SD of the 29 eyes which were able to be measured by both the $78 \mathrm{D}$ lens and the optic disc biometer, are detailed in Table 1. The measurements made by the $78 \mathrm{D}$ lens have been multiplied by 1.16 (as the magnification factor for the lens is $0 \cdot 86$ ).

\section{MEASUREMENT OF OPTIC DISC SIZE FROM THE} PROJECTED SLIDES

From a scattergram plot of the two observers' measurements of vertical optic disc diameter of the 29 eyes measured, the regression line $y=0.983 x+0.2712$ is calculated. This shows a very strong correlation $r=0.970 \quad(p<0.0001)$ between the two observers. Agreement was examined by plotting the difference between

Table 1 Optic disc size compared for the three photographic estimates with the $78 \mathrm{D}$ lens and biometer measurements ( $\mathrm{mm})$

\begin{tabular}{lllll}
\hline Method & $\begin{array}{l}\text { Smallest } \\
\text { disc }\end{array}$ & $\begin{array}{l}\text { Largest } \\
\text { disc }\end{array}$ & $\begin{array}{l}\text { Mean of } \\
29 \text { eyes }\end{array}$ & $\begin{array}{l}\text { SD of } \\
29 \text { eyes }\end{array}$ \\
\hline Estimate 1 & 1.335 & 2.441 & 1.736 & 0.210 \\
Estimate 2 & 1.374 & 2.276 & 1.740 & 0.185 \\
Estimate 3 & 1.365 & 2.388 & 1.709 & 0.205 \\
78 D lens & 1.48 & 2.53 & 2.119 & 0.237 \\
Biometer & 1.249 & 2.311 & 1.860 & 0.249 \\
\hline
\end{tabular}


Table 2 The correlation for each of the three photographic estimates with the $78 \mathrm{D}$ lens and biometer measurements, for the 23 eyes within plus or minus $3 \mathrm{D}$ and all 29 eyes

\begin{tabular}{|c|c|c|c|c|c|}
\hline \multirow[b]{2}{*}{$\begin{array}{l}\text { Planimetric } \\
\text { estimate }\end{array}$} & \multirow[b]{2}{*}{$\begin{array}{l}\text { Indirect } \\
\text { method }\end{array}$} & \multicolumn{2}{|l|}{23 Eyes } & \multicolumn{2}{|l|}{29 Eyes } \\
\hline & & $\begin{array}{l}\text { Correlation } \\
\text { coefficient } r\end{array}$ & $p$ Value & $\begin{array}{l}\text { Correlation } \\
\text { coefficient } r\end{array}$ & p Value \\
\hline \multirow[t]{2}{*}{ Estimate 1} & $78 \mathrm{D}$ lens & 0.5449 & 0.0059 & 0.4178 & 0.0216 \\
\hline & Biometer & 0.3639 & 0.0805 & 0.2936 & $0 \cdot 1153$ \\
\hline \multirow{2}{*}{ Estimate 2} & $78 \mathrm{D}$ lens & 0.6890 & 0.0002 & 0.5378 & 0.0022 \\
\hline & Biometer & 0.5431 & 0.0061 & 0.4301 & 0.0178 \\
\hline \multirow[t]{2}{*}{ Estimate 3} & $78 \mathrm{D}$ lens & 0.6922 & 0.0002 & 0.5286 & 0.0027 \\
\hline & Biometer & 0.5604 & 0.0044 & 0.4207 & 0.0205 \\
\hline
\end{tabular}

the two measurements against the mean. Observer A did not read consistently higher or lower than observer B at any size of optic disc as the slope of the regression line is not significant $(r=0.065, \mathrm{p}=0.735)$. There is also no constant difference as the regression line $(y=0 \cdot 250 x+16 \cdot 64)$ passes through zero at the overall mean optic disc size. There is no significant difference between the two observers' measurements by a two tailed paired Student's $t$ test $(p=0.931)$. The mean of the measurements was therefore used in further calculations.

\section{COMPARISON WITH BENGTSSON AND KRAKAU'S} CALCULATIONS

The three estimates were then applied to the mean image height obtained from the photographic slides. The mean, SD, and range of optic disc size obtained are compared with the $78 \mathrm{D}$ lens and biometer measurements in Table 1 . The $78 \mathrm{D}$ lens measurements and the biometer measurements were plotted against the photographic measurements, using each of the three estimates, and simple regression analysis performed (Table 2).

Estimate 1 based on ultrasonography

The $78 \mathrm{D}$ lens measurements were significantly different from the photographic measurements by a paired two tailed Student's $t$ test $(p<0.0001)$. There was also a significant difference between the biometer and photographic measurements by the same test $(p=0.0218)$.

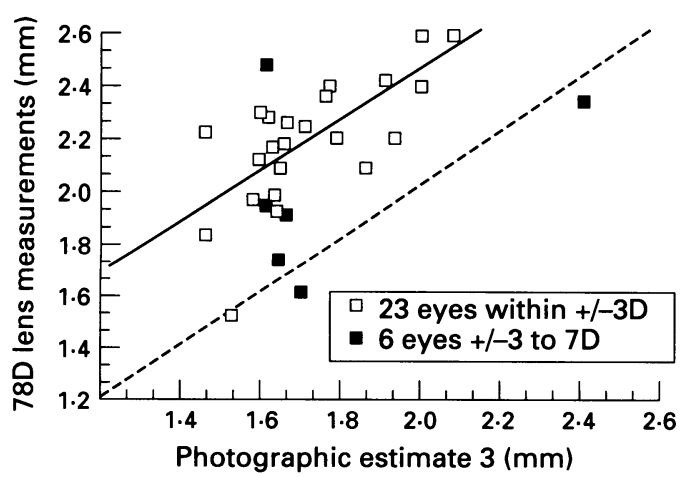

Figure $178 \mathrm{D}$ lens measurements of optic disc size are compared with the photographic measurements calculated using estimate 3. The regression line for the 23 eyes within plus or minus $3 \mathrm{D}$ of emmetropia is shown. The line of identity is also shown.
Estimate 2 based on spectacle refraction

The $78 \mathrm{D}$ lens measurements were significantly different from the photographic measurements by a paired two tailed Student's $t$ test $(\mathrm{p}<0.0001)$. There was also a significant difference between the biometer and photographic measurements by the same test $(\mathrm{p}=0.0112)$.

Estimate 3 based on refraction and keratometry

The results of the $78 \mathrm{D}$ lens measurements by observer 1 and the photographic measurements are compared in Figure 1. The biometer measurements by observer 2 are compared in Figure 2. The best correlation between the readings is found with this photographic estimate and the $78 \mathrm{D}$ lens $(r=0.692, \mathrm{p}=0.0002$ for regression line $y=0.951 x+0.543)$ for the 23 eyes within plus or minus $3 \mathrm{D}$ of emmetropia.

As before the $78 \mathrm{D}$ lens measurements were significantly different from the photographic measurements by a paired two tailed Student's $t$ test $(\mathrm{p}<0.0001)$. There was also a highly significant difference between the biometer and photographic measurements by the same test $(p=0.0027)$.

For the six eyes with a higher refractive error there was a greater disparity between the photographic and $78 \mathrm{D}$ lens measurements than for the 23 eyes within plus or minus 3 dioptres of emmetropia. A Mann-Whitney $U$ test showed this to be highly significant $(p=0.0001)$. Similarly the disparity between the photographic and biometer measurements was significantly greater for the six eyes with high refractive errors $(p<0.0001)$.

Agreement can also be examined by plotting the difference between the vertical disc diameter calculated by the photographic method (using estimate 3 ) and the $78 \mathrm{D}$ lens against the photographic measurements (Fig 3). Overall, the $78 \mathrm{D}$ lens measures larger than the photographic method by a mean of $0.41 \mathrm{~mm}$, taking into account the magnification factor for the lens $(\times 0.86)$. There is a tendency for the $78 \mathrm{D}$ lens to further overestimate the size of the smaller discs, but the slope of the regression line $(y=0.263 x+1.81, r=0.299)$ is not significant $(p=0 \cdot 109)$. Nineteen of the

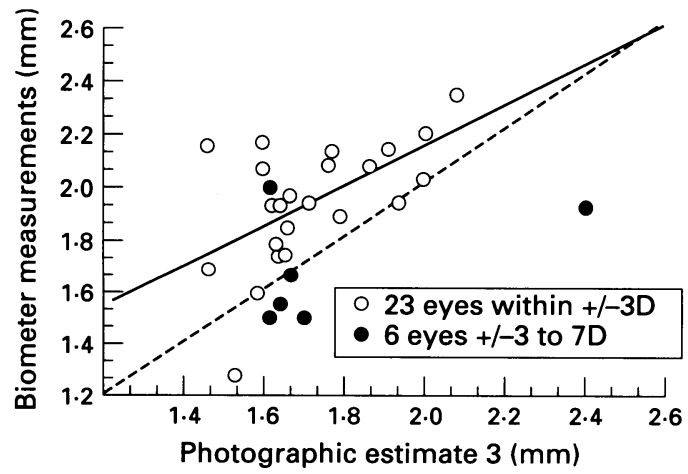

Figure 2 Biometer measurements of optic disc size are compared with the photographic measurements calculated using estimate 3. The regression line for the 23 eyes within plus or minus $3 \mathrm{D}$ of emmetropia is shown. The line of identity is also shown. 


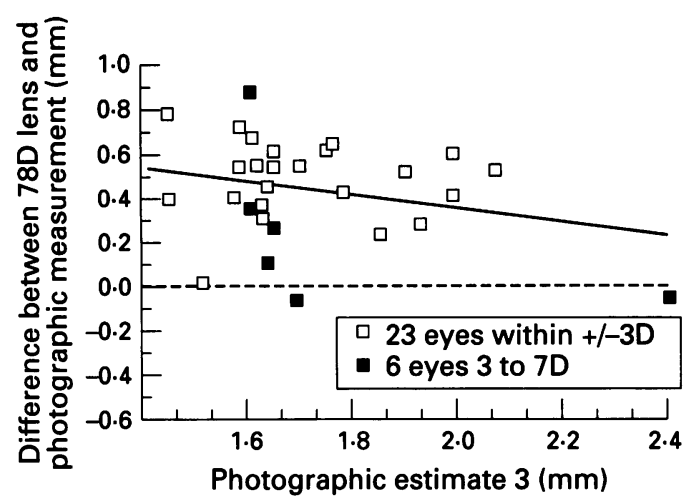

Figure 3 Agreement between the optic disc size measured by the $78 \mathrm{D}$ lens and by photographic estimate 3 . The difference between the two measurements is plotted against the photographic measurement for each of the 29 eyes. The regression line is shown.

measurements lie outside the mean difference plus or minus $0.1 \mathrm{~mm}(63 \%)$ and nine lie outside the mean plus or minus $0.2 \mathrm{~mm}(30 \%)$. If the magnification factor is not applied the $78 \mathrm{D}$ lens measurements are still larger by a mean of $0.12 \mathrm{~mm}$ and differ significantly by paired Student's $t$ test $(\mathrm{p}=0.0047)$ from the planimetric measurements. Correlation is unchanged and when agreement is examined the scatter of results is also unchanged.

Agreement between the optic disc biometer and photographic measurements (using estimate 3 ) is demonstrated similarly in Figure 4. The biometer measures, on average, $0.15 \mathrm{~mm}$ larger than the photographic method. The regression line $(y=0 \cdot 166 x+1 \cdot 760, r=0 \cdot 214)$ also shows a tendency for the biometer to further overestimate the size of the smaller optic discs but this is not significant $(p=0.2571)$. Twenty of the measurements lie outside the mean difference plus or minus $0.1 \mathrm{~mm}(69 \%)$ and 11 lie outside the mean difference plus or minus $0.2 \mathrm{~mm}$ $(38 \%)$.

\section{$78 \mathrm{D}$ lens and optic disc biometer measurements compared}

There was a stronger correlation between the $78 \mathrm{D}$ lens and biometer measurements $(r=0.870, \mathrm{p}<0.0001)$ than between either clinical method and the photographic measurements for all 29 eyes. If the two methods are compared by a paired two tailed Student's $t$ test they show significantly different measurements $(p<0.0001)$. Over all optic disc sizes, the biometer measures smaller than the $78 \mathrm{D}$ lens, the regression line $(y=0.250 x+1.925, r=0.133)$ is not significant $(p=0.485)$ showing that the difference does not vary significantly as disc size increases.

\section{Intraobserver variation for $78 \mathrm{D}$ lens measurements}

The coefficient of variation was calculated as the square root of the mean value of the variance of the measurements taken three times for each of the 29 optic discs and then divided by the mean measured disc diameter.

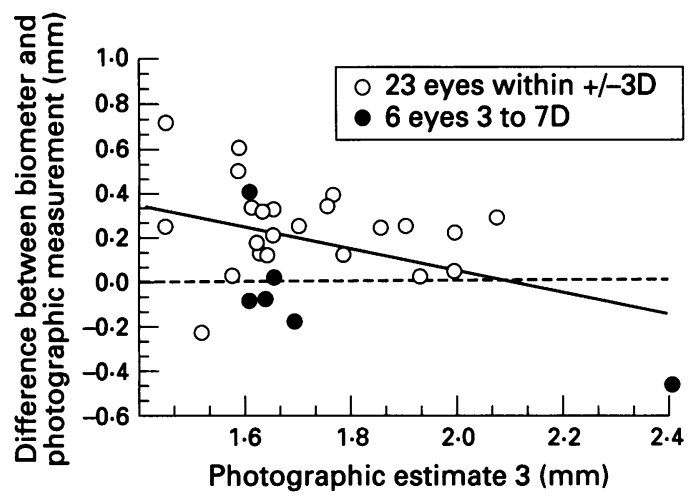

Figure 4 Agreement between the optic disc size measured by the optic disc biometer and by photographic estimate 3. The difference between the two measurements is plotted against the photographic measurement for each of the 29 eyes. The regression line is shown.

As the mean variance is 0.0027 and the mean measured disc size is $2.119 \mathrm{~mm}$, the coefficient of variation is 0.0245 .

\section{Intraobserver variation for optic disc biometer measurements}

The coefficient of variation was calculated as above using the mean value of the variance of the measurements taken three times for each of the 29 optic discs and then divided by the mean measured disc diameter.

As the mean variance is 0.0034 and the mean measured disc size is $1.860 \mathrm{~mm}$, the coefficient of variation is 0.0313 .

\section{Discussion}

Both of the above clinical methods of estimating optic disc size share the same optical principle, that of indirect ophthalmoscopy. A real aerial inverted image is formed and this is viewed either, if using the $78 \mathrm{D}$ lens, by focusing the slit-lamp biomicroscope, or the image falls on the screen at the principal plane of the condensing lens, if using the optic disc biometer. It would therefore be expected that both methods would yield similar readings in individual eyes. However, this study shows that, in our hands, the two methods differ significantly from each other and from established planimetric techniques.

The $78 \mathrm{D}$ lens measurements were, on average, $0.41 \mathrm{~mm}$ larger and the biometer measurements $0.15 \mathrm{~mm}$ larger than the third photographic estimate which uses spectacle refraction and corneal curvature. The closest correlation between the planimetric and clinical methods was found using this photographic estimate as noted in previous studies of optic disc size estimation. ${ }^{24} 25$ As the $78 \mathrm{D}$ lens measurements are so much larger than planimetry when corrected with the manufacturer's magnification factor $(0.86)$ this suggests that their calculations for the correction factor are not correct when the lens is used at the Haag-Streit slit-lamp biomicroscope. If the uncorrected measurements are used the $78 \mathrm{D}$ lens measures $0.12 \mathrm{~mm}$ larger on average and correlation with planimetry is unchanged. Correlation alone only shows the strength of 
the relation between the measurements, not the agreement. Our results differ from a recent publication $^{22}$ in which the $90 \mathrm{D}$ lens was used to estimate optic disc size at the slit-lamp, where a higher correlation with planimetric techniques was obtained. Both methods used in this study demonstrate marked scatter of the results when agreement is examined ${ }^{26}$ (as in Figs 3 and 4) and surprisingly a substantial number of measurements were outside plus or minus $0.2 \mathrm{~mm}$ from the mean difference between the measurements $(30 \%$ for the $78 \mathrm{D}$ lens and $34 \%$ for the biometer). The biometer and $78 \mathrm{D}$ lens appeared to further overestimate the size of the smaller discs but this was not statistically significant. This factor cannot be compared with the $90 \mathrm{D}$ lens study as agreement (see above) was not assessed by the author. It would be interesting to see the data from that study replotted as it is likely that the $90 \mathrm{D}$ lens also overestimates the smaller optic discs.

Previous authors have described both direct and indirect ophthalmoscopic methods of optic disc analysis and the use of the Goldmann fundus contact lens at the slitlamp. ${ }^{2728}$ The latter method required a graticule ${ }^{27}$ or a calculated correction factor which varied with the refraction of the eye. ${ }^{28}$ No magnification constant was calculated for the contact lens and the 'normal range' of optic discs measured differed from that described by planimetric and histological studies. ${ }^{89}$ Increasing refractive error is known to cause inaccuracies in both direct and indirect methods. $^{27}$ Refractive error influences the image size produced if the focal plane of the condensing lens does not coincide with the anterior focus of the eye. At the slit-lamp biomicroscope with the $78 \mathrm{D}$ lens, the image obtained may appear 'focused' adequately to take a reading with the slit-beam, when in fact the lens is not exactly at the anterior focus of the eye. This may account for some of the disparity between the readings obtained with the $78 \mathrm{D}$ lens and planimetry. However, for our observer the method appears highly repeatable as there was a low coefficient of variation. As the $78 \mathrm{D}$ lens measures greater even in the emmetropic eye this suggests that the disparities are not merely in the position of the condensing lens but a correction factor for the magnification of the eye may need to be calculated for eyes of greater refractive error. This is supported by the significantly greater disparity found between the measurements for the six eyes with higher refractive errors. The small number of eyes of higher refractive error examined in this study does not permit the calculation of correction factors for increasing ametropia. Many glaucoma suspects are myopic and although the majority will not have marked ametropia this will restrict the use of indirect ophthalmoscopic methods to estimate disc diameter in these patients.

Montgomery in his report of the optic disc biometer ${ }^{1921}$ explained the importance of the positioning of the optical spacer. The optical spacer (which incorporates the condensing lens) needs to be held steady at a distance from the patient's eye where the best focus is achieved; it requires the operator to have a large hand span to facilitate this manoeuvre. It is quite probable that the different results achieved with the instrument in comparison with planimetry are due to the lens being moved in relation to the anterior focus of the eye. The technique also requires coordination of the optical spacer, the digitised calipers, and the footswitch to take a measurement and therefore is not as simple to perform as the $78 \mathrm{D}$ lens measurement at the slit-lamp. With increasing ametropia we would expect the disparity between the biometer and planimetric results to increase and indeed our findings confirm this. However, this does not account for all the disparity with planimetry as we might expect that operator error would induce a marked intraobserver variation and this was not the case. It should also be noted that no previous publication on the biometer compares it with established planimetric techniques.

Assessment of the size of the optic disc, even by more sophisticated techniques, relies on the interpretation of the optic disc boundary. Although the two observers in this study used two different techniques to measure the optic disc diameter both observers were experienced in optic disc assessment from previous studies. ${ }^{24} 25$ In addition, they had previously shown very little interobserver variability in identifying the optic disc boundary ${ }^{24}$ so it would seem unlikely that the differences between the planimetric and clinical methods were due to great variability between the observers' recognition of the optic disc boundary. The optic disc biometer itself has been assessed previously for interobserver variability $^{29}$ and median interobserver difference was shown to be $4 \cdot 45 \%$. The observer using the $78 \mathrm{D}$ lens showed a low intraobserver variability similar to that found by the same observer using the Zeiss four mirror contact lens to measure disc diameter ${ }^{25}$ and also consistent with the previous study published using the $90 \mathrm{D}$ lens. ${ }^{22}$ Although a small error caused by interobserver variability of the clinical methods will affect the calculations of correlation and agreement this alone would not account for the relatively poor correlation and agreement between the indirect ophthalmoscopic and planimetric measurements.

Further inaccuracies may be due to the planimetric techniques. Bengtsson and Krakau's previous calculations ${ }^{30}$ have been shown to be equivalent to Littman's algorithms to correct for the magnification factor of the eye, when using the Zeiss fundus camera. ${ }^{31}$ This study uses their most recent planimetric corrections ${ }^{23}$ which have been shown to correlate well with disc diameter measured in vivo with the Zeiss four mirror contact lens ${ }^{25}$ and with the Heidelberg retina tomograph (HRT) confocal scanning laser ophthalmoscope. ${ }^{24}$ The HRT measurements were smaller than the planimetric measurements, if the size difference is corrected for then good agreement is achieved. All planimetric methods will include error as at present they cannot be verified reliably in living eyes. Optic disc size calculations 
using Littman's formula were shown to differ from measurements during vitrectomy. ${ }^{32}$ Other investigators have demonstrated that decentration of the object, alteration of the eye to camera distance, and increasing ametropia can cause a large variation in the measured from the calculated magnification. ${ }^{33-35}$ However, planimetric calculations are at present the 'gold standard' with which new methods of optic disc measurement are compared.

The $78 \mathrm{D}$ lens and optic disc biometer techniques both appeared to be repeatable with a coefficient of variation of $2.45 \%$ and $3.13 \%$ respectively. This is comparable with the $3.07 \%$ calculated from the figures given in the $90 \mathrm{D}$ lens study. ${ }^{22}$ Intraobserver variation has been assessed for the optic disc biometer previously as $2 \cdot 7 \% .{ }^{19}$ Both methods, therefore, show an acceptable low variability for a clinical technique of measurement.

Of the two techniques employed in this study, the $78 \mathrm{D}$ lens is simpler and shows a better correlation but poorer agreement with planimetry. Better agreement may, however, be achieved by eliminating the manufacturer's magnification factor from the calculations as this reduces the mean difference between the $78 \mathrm{D}$ lens and planimetry to $0.12 \mathrm{~mm}$. It may be a useful technique for a rapid assessment of whether the optic disc is unusually large or small, providing that the clinician knows the normal range of disc size for this method. Alternatively, extrapolating from our results, we suggest that an approximation of optic disc diameter can be obtained by dividing the disc size, measured with the $78 \mathrm{D}$ lens, by 0.86 and subtracting $0.41 \mathrm{~mm}$. One must remember that, after this calculation, there is only likely to be a $70 \%$ chance of the result obtained being within plus or minus $0.2 \mathrm{~mm}$ of the planimetric calculation, based on the results of an experienced observer.

We wish to thank Mr Ahmed Sadiq for his help with measurement of the vertical optic disc diameter from photographic slides and Mr Peter Pawson for his help with clinical dat collection.

1 Jonas JB, Gusek GC, Naumann GOH. Optic disc morphometry in chronic primary open-angle glaucoma I. Morphometric intrapapillary characteristics. Graefes Arch Clin Exp Ophthalmol 1988; 226: 522-30.

2 Yablonski ME, Zimmeman TJ, Kass MA, Becker B. Prognostic significance of optic disc cupping in ocular Prognostic significance of optic disc cupping in ocular
hypertensive patients. Am $\mathcal{f}$ Ophthalmol 1980; 89: 585-92.

3 Tuulonen A, Airaksinen PJ. Initial glaucomatous optic disk and retinal nerve fibre layer abnormalities and their progression. Am $\mathcal{F}$ Ophthalmol 1991; 111: 485-90.

4 Pederson JE, Anderson DR. The mode of progressive disc cupping in ocular hypertension and glaucoma. Arch Ophthalmol 1980; 98: 490-5.

5 Sommer A, Polack I, Maumenee E. Optic disc parameters and onset of glaucomatous field loss 1 . Methods and progressive changes in disc morphology. Arch Ophthalmol 1979; 97: 1444-8.

6 Caprioli J, Miller JM, Sears M. Quantitative evaluation of the optic nerve head in patients with unilateral visual field loss from primary open-angle glaucoma. Ophthalmology 1987; 94: 1484-7.

7 Varma R, Steinman WC, Scott IU. Expert agreement in evaluating the optic disc for glaucoma. Ophthalmology 1992; 99: 215-21.

8 Jonas JB, Gusek GC, Guggenmoos-Holzmann I, Naumann GOH. Variability of the real dimensions of normal human optic discs. Graefes Arch Clin Exp Ophthalmol 1988; 226: optic discs

9 Quigley HA, Brown AE, Morrison JD, Drance SM. The size and shape of the optic disc in normal human eyes. Arch Ophthalmol 1990; 108: 51-7.

10 Heijl A, Molder H. Optic disc diameter influences the ability to detect glaucomatous optic disc damage. Arch Ophthalmol (Copenh) 1993; 71: 122-9.

11 Bengtsson B. The variation and covariation of cup and disc diameters. Arch Ophthalmol (Copenh) 1976; 54: 804-18.

12 Jonas JB, Gusek GC, Naumann GOH. Optic disc, cup and neuroretinal rim size, configuration and correlations in neuroretinal rim size, configuration and correlations in

13 Balazsi AG, Drance SM, Schulzer M, Douglas GR. Neuroretinal rim area in suspected glaucoma and early chronic open-angle glaucoma. Arch Ophthalmol 1984; 102: $1011-4$.

14 Takamoto T, Schwartz B. Reproducibility of photogrammetric optic disc cup measurements. Invest Ophthalmol Vis Sci 1985; 26: 814-7.

15 Schwartz B. New technique for the examination of the optic disc and their clinical application. Trans Am Acad disc and their clinical application. Tran
Ophthalmol Otolaryngol 1976; 81: 227-35.

16 Portney GL. Photogrammetric analysis of volume asymmetry of the optic nerve head cup in normal, hypertensive and glaucomatous eyes. Am F Ophthalmol 1975; 80: 51-5.

17 Dandona L, Quigley HA, Jampel HD. Variability of depth measurements of the optic nerve head and peripapillary retina with computerized image analysis. Arch Ophthalmol 1989; 107: 1786-92.

18 Caprioli J, Klingbeil U, Sears M, Pope B. Reproducibility of optic disc measurements with computerized analysis of stereoscopic video images. Arch Ophthalmol 1986; 104: 1035-9.

19 Montgomery DMI. Measurement of optic disc and neuroretinal rim areas in normal and glaucomatous eyes a new clinical method. Ophthalmology 1991; 98: 50-9.

20 Montgomery DMI. Clinical disc biometry in early glaucoma. Ophthalmology 1993; 100: 52-6.

21 Montgomery DMI. The optical spacer - a simple device which extends the scope of indirect ophthalmoscopy. Br f Ophthalmol 1992; 76: 45-6.

22 Ruben S. Estimation of optic disc size using indirect biomicroscopy. Br f Ophthalmol 1994; 78: 363-4.

23 Bengtsson B, Krakau CET. Correction of optic disc measurements on fundus photographs. Graefes Arch Clin Exp Ophthalmol 1992; 230: 24-8.

24 Spencer AF, Vernon SA. Vertical optic disc diameter - the Heidelberg Retina Tomograph against photographs. Invest Ophthalmol Vis Sci 1995; 36: 796-803.

25 Spencer AF, Vernon SA. Optic disc measurement with the Zeiss 4-mirror contact lens. Brf Ophthalmol 1994; 78: 775-80.

26 Bland JM, Altman DG. Statistical methods for assessing agreement between two methods of clinical measurement. Lancet 1986; i: 307-10.

27 Franceschetti A, Bock RH. Megalopapilla: a new congenital anomaly. Am ₹ Ophthalmol 1950; 33: 227-34.

28 Beuchat L, Safran AB. Optic nerve hypoplasia: papillary diameter and clinical correlation. $\mathcal{f}$ Clin Neuro-ophthalmol 1985; 5: 249-53.

29 Pyott AAE, Montgomery DMI. Inter-observer variation in clinical optic disc biometry. Eye 1993; 7: 452-6.

30 Bengtsson B, Krakau CET. Some essential optical features of the Zeiss fundus camera. Arch Ophthalmol 1977; 55: 123-31.

31 Mansour AM. Measuring fundus landmarks. Invest Ophthalmol Vis Sci 1990; 31: 41-2.

32 Bartz-Schmidt KU, Weber J, Heinmann K. Optic disc size calculations using Littmann's formula differs from in vivo measurements of the optic disc during vitrectomy. Invest measurements of the optic disc during

33 Pach J, Pennell DO, Romano PE. Optic disc photogrammetry: magnification factors for eye position, centration, and ametropias, refractive and axial; and their application in the diagnosis of optic nerve hypoplasia. Ann Ophthalmol 1989; 21: 454-62.

34 Lotmar W. Dependence of magnification upon the camerato-eye distance in the Zeiss fundus camera. Arch Ophthalmol 1984; 62: 131-4.

35 Arnold JV, Gates JWC, Taylor KM. Possible errors in the measurement of retinal lesions. Invest Ophthalmol Vis Sci 1993; 34: 2576-80. 